\title{
Effects of Steam Explosion Processing on Quality of Red Beans
}

\author{
Li Chao-lu-meng, Zhang Xue-jin, Chen Kun-jie* \\ College of Engineering, Nanjing Agricultural University, Nanjing, China
}

Email address:

lichaolumeng@126.com (Li Chao-lu-meng), kunjiechen@njau.edu.cn (Chen Kun-jie)

${ }^{*}$ Corresponding author

\section{To cite this article:}

Li Chao-lu-meng, Zhang Xue-jin, Chen Kun-jie. Effects of Steam Explosion Processing on Quality of Red Beans. International Journal of Nutrition and Food Sciences. Vol. 6, No. 3, 2017, pp. 129-133. doi: 10.11648/j.ijnfs.20170603.13

Received: February 26, 2017; Accepted: March 20, 2017; Published: April 7, 2017

\begin{abstract}
Steam explosion pressure, holding time and initial moisture content of red beans were used as variables for the experimental design, according to BBD (Box-Behnken) central composite model. Red beans were processed in steam explosion and the changes of protein, fat, cellulose and other nutritive contents of red beans, changes of hardness, springiness, chewiness and other texture properties, and changes of puffing ratio and cooking properties after the steam explosion processing were determined to analyze the effects of steam explosion processing on quality of red beans. After the processing, the content of crude protein, crude fat and crude cellulose decreased by $0.84 \%, 0.02 \%$ and $0.33 \%$ respectively, while the content of starch increased by $0.58 \%$, but no significant effect of steam explosion processing on nutritive contents of red beans was identified $(P>0.05)$; after the steam explosion processing, the average springiness and chewiness increased by $0.15 \mathrm{~mm}$, $1.07 \mathrm{~mJ}$ respectively, but no significant difference with that before such processing was identified $(P>0.05)$. However, the hardness reduced by $22.0 \mathrm{~N}$ with a very significant change $(P<0.01)$; through the steam explosion processing, the puffing ratio of red beans increased by $95.4 \%$ while the cooking time of bean soup shortened by $50 \%$, with a very significant change $(P<0.01)$.
\end{abstract}

Keywords: Red Beans, Steam Explosion, Puffing, Quality

\section{Introductions}

The Red bean also known as Azuki Bean (small red bean or small bean) with significant medicinal and nutritional value is one of the major grains in the northern part of China [1]. Moreover, With the social development and improvement of living standards, there is a growing demand for food convenience, quality and nutrition, but the conventional puffed food cannot fully meet this demand anymore, the research and development of convenient, nutritional and green puffed food gradually becomes the evolution orientation of puffed food $[2,3]$. Red bean is often used as the raw material for cooking congee (one of traditional Chinese food) due to the significant nutritional value. However, when red beans are cooked to prepare red bean congee, red bean paste and other food, the cooking process will be time and energy-consuming and it is tough to soften red beans, due to the compact structure of seed coat and low water absorption. To address these problems, people often adopt high pressure cooking, $\mathrm{Na}_{2} \mathrm{CO}_{3}$-added cooking or other methods, but high pressure cooking has a higher requirement for the cooking equipment, while $\mathrm{Na}_{2} \mathrm{CO}_{3}$-added cooking process can reduce the cooking time, it may significantly damage the nutritive contents of red beans. Therefore, high pressure cooking or $\mathrm{Na}_{2} \mathrm{CO}_{3}$-added cooking is not the appropriate processing method for red beans.

Steam explosion is a material pretreatment method which has been rapidly developing over these years. It processes the material in explosion through instantaneous pressure release after holding the biomass material under high temperature and high pressure. After processing, the material has a fluffy texture, the contact area between substrate and enzyme significantly enlarges, which rapidly increases the yield of extracts and considerably shortens the cooking time $[4,5]$. 
Therefore, if red beans are appropriately pretreated in steam explosion using steam explosion method, red beans will be properly puffed, the epidermal tissue appears a certain change, the structure becomes fluffy, but the basic morphologies and nutritive contents of red beans are kept undamaged. If a consumer cooks red bean soup or prepares red bean paste using puffing pretreatment, red beans can be easily processed with convenience and energy efficiency [6]. By now, no domestic or foreign work has observed the puffing pretreatment on red beans through steam explosion method. This study attempts to look into the changes of nutrients, texture and cooking properties after the steam explosion processing through experimental method, which will provide a theoretical and technical basis for subsequent research and development of the pre-puffed food made of red beans.

\section{Materials and Methods}

\subsection{Materials and Reagents}

The experimental red beans were grown in Baoqing County of Heilongjiang, subpackaged in Nanjing, and purchased from a local supermarket - Suguo.

Reagents include concentrated sulfuric acid, copper sulfate pentahydrate, potassium hydroxide, sodium hydroxide, hydrochloric acid, boric acid, tracer agent, ammonium sulfate, petroleum ether (boiling point: $30 \sim 60^{\circ} \mathrm{C}$ ), sulfuric acid, potassium hydroxid, decalin, ethanol, iodine solution, amylas, toluene, DNS displaying solution, sea sand. These are analytical reagents (Ars).

\subsection{Instrument and Equipment}

QBS-80B Steam Explosion Test Bench, supplied by Hebi Zhengdao Biological Energy Co., Ltd; DHG-10 Oven, supplied by Shanghai Hualian Medical Equipment Co., Ltd; JFSO-100 Grinder, supplied by Shanghai Jiading Food Oil Instrument Co., Ltd; SH220K Graphite Digestion Instrument, supplied by Jinan Hannon Instrument Co., Ltd; K9860 Automatic Kjeldahl Analyzer, supplied by Jinan Hannon Instrument Co., Ltd; SOX500 Automatic Fat Analyzer, supplied by Jinan Hannon Instrument Co., Ltd; FIWE6 Cellulose Tester, supplied by Italian VELP; SHZ-D (III) Circulating Water Pump, supplied by Gongyi Yu Hua Instrument Co., Ltd; TMS-PRO Texture Analyzer, supplied by US FTC; CFXB40FZ12-85 SUPOR Luxury Intelligent Electric Cooker, supplied by Zhejiang Supor Co., Ltd; 759S Ultraviolet and Visible Spectrophotometer, supplied by Shanghai Jinghua Technology Instrument Co., Ltd; FA1004 Electronic Balance, supplied by Shanghai Sunny Hengping Science Instrument Co., Ltd; $1 \mathrm{~cm}, 5 \mathrm{~cm}$ sieve, supplied by Zhejiang Shaoxing Huafeng Experiment Instrument Co., Ltd.

\subsection{Experimental Design}

The levels of these three factors were recognized through experiment, and the code and levels of independent factors were designed in accordance with Box-Behnken (BBD) model $[7,8]$, as shown in Table 1:

Table 1. Levels and code of independent factors.

\begin{tabular}{llll}
\hline Level & Factor & & \\
\hline-1 & 0.4 & 120 & 15 \\
0 & 0.6 & 220 & 20 \\
1 & 0.8 & 320 & 25 \\
\hline
\end{tabular}

\subsection{Experimental Methods}

\subsubsection{Steam Explosion Experiment}

Fresh and healthy red beans with intact coat were selected and filtered through $5 \mathrm{~cm}$ grain sieve; the moisture content of red beans was measured as required by initial moisture content, about $1,000 \mathrm{~g}$ (approximately $1 / 2$ of the steam explosion chamber volume) red beans after soaking were picked for each group and placed in the steam explosion chamber for carrying out the experiment; after the steam explosion, red beans were immediately transferred to the oven for drying until they met the standard moisture level of beans safety storage [9,10], removed and cooled down to the room temperature, then wrapped in the bag and stored at $4-8^{\circ} \mathrm{C}$. Each experiment was repeated for three times and the results of three experiments were averaged for the analysis.

\subsubsection{Nutritive Contents}

Nitrogen content of red beans was determined and crude protein content was calculated using Kjeldahl method, and the specific procedures were with reference to $\mathrm{GB} / \mathrm{T}$ 5511-2008/ISO 20483:2006. Crude fat content of red beans was determined using Soxhlet extraction and the specific procedures were with reference to GB/T 14772-2008. Starch content was determiend using enzyme hydrolysis among GB determination methods and the specific procedures were with reference to GB5009.9-85. Crude cellulose content of red beans was determiend using medium filtration as contained in "Food Crude Cellulose Content Determination", and the specific operations were with reference to $\mathrm{GB} / \mathrm{T}$ 5515-2008/ISO 6865:2000.

\subsubsection{Puffing Ratio}

Puffing ratio of red beans was determined using quartz sand volume determination method [11].

\subsubsection{Texture Properties}

The samples consist of 50 grains were selected from unprocessed red beans and explosion red beans, and TPA (explan) test was carried through texture analyzer. Cylindrical probes were used and test parameters were set as: induction force: $0.5 \mathrm{~N}$, initial force: $0.1 \mathrm{~N}$, test rate: $60 \mathrm{~mm} / \mathrm{s}$, compression deformation: $50 \%[12,13]$.

\subsection{Statistical Analysis}

Each experiment was repeated for three times and data analysis was done by SPSS19.0 and Excel2010. 


\section{Results and Analysis}

\subsection{Effects of Steam Explosion Processing on the Nutritive Content}

\subsubsection{Effects of Steam Explosion Processing on the Crude Protein Content}

Table 2. Crude protein content statistics of the explosion red beans.

\begin{tabular}{lrrrrc}
\hline Raw red beans & 17.59 & 19.03 & 18.41 & 0.808 & 0.374 \\
Explosion red beans & 14.28 & 20.76 & 17.57 & & \\
\hline $\begin{array}{l}\text { Note: ** means } \mathrm{P}<0.01 \text {, very } \\
\text { P significant; }\end{array}$ * means, insignificant & $\mathrm{P}<0.05$, significant; \\
\end{tabular}

Table 2 shows the statistical analysis of crude protein content of unprocessed beans and exploded red beans. as it can be seen from Table 2 the crude protein content of red beans as a whole decreased to some extent, with an average decrease of $0.84 \%$, but no significance difference was identified. However, the effects on crude protein content of red beans may vary under different steam explosion conditions. the Fig. 1 shows the changes of crude protein content of red beans under different steam explosion conditions. Analysis of variance suggests that in contrast with unprocessed red beans, only the red beans processed under No.7 experiment condition experienced a significant decrease of crude protein content, while other processing conditions showed in non-significant effects on crude protein content of red beans. This indicates that the steam explosion processing has no significant effect on the protein content of red beans.

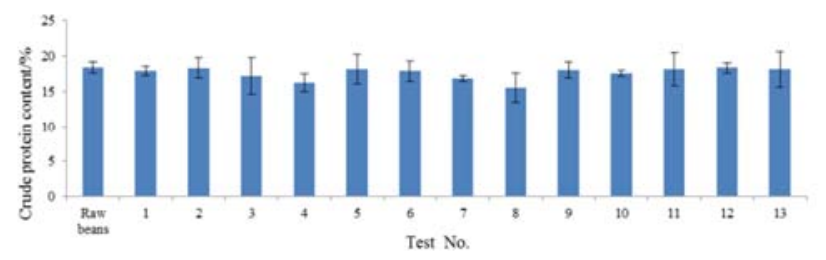

Figure 1. Crude protein content of explosion beans and unprocessed red beans.

\subsubsection{Effects of Steam Explosion Processing on Crude Fat Content}

Table 3. Variance analysis of crude fat content of explosion red beans.

\begin{tabular}{llllll}
\hline Raw red beans & 0.58 & 0.82 & 0.69 & 0.085 & 0.772 \\
Explosion red beans & 0.49 & 0.87 & 0.67 & & \\
\hline
\end{tabular}

Note: ** means $\mathrm{P}<0.01$, very significant; * means $\mathrm{P}<0.05$, significant; $\mathrm{P}>0.05$, insignificant.

Table 3 shows the statistical anlaysis of crude fat content of unprocessed beans and explosion red beans, and Fig. 2 presents the crude fat content of red beans under different experimental conditions. As it can be seen from Table 3 the average crude fat content of red beans only decreased by $0.02 \%$, basically with no change. Analysis of variance suggests that, while the crude fat content of red beans varied under different experimental conditions, such changes were insignificant. This indicates that the steam explosion processing has non-significant effect on the fat content of red beans.

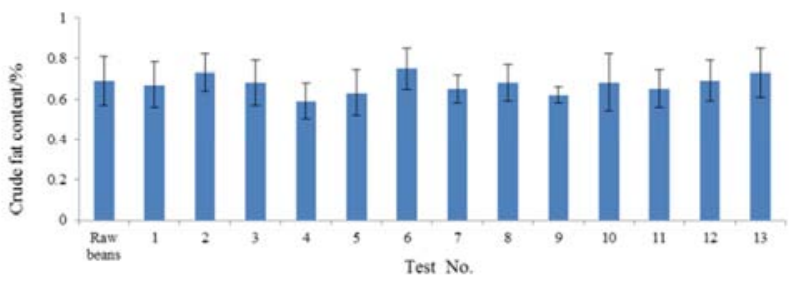

Figure 2. Crude fat content of explosion red beans and unprocessed beans.

\subsubsection{Effects of Steam Explosion Processing on the Starch Content}

Table 4. Variance analysis of starch content statistics of the explosion red beans.

\begin{tabular}{llllll}
\hline Raw red beans & 37.65 & 42.70 & 40.10 & 0.150 & 0.701 \\
Explosion red beans & 36.09 & 47.22 & 40.68 & & \\
\hline
\end{tabular}

Note: ** means $\mathrm{P}<0.01$, very significant; * means $\mathrm{P}<0.05$, significant; $\mathrm{P}>0.05$, insignificant.

Table 4 shows the statistical analysis of starch content of unprocessed beans and explosion red beans, as it can be seen from Table 4, the average starch content of red beans increased by $0.58 \%$, with insignificant average change. According to the starch content results (see Fig.3) of red beans under different experimental conditions, after the steam explosion processing under higher pressure and through longer holding time, the starch contents of explosion red beans were higher than that of unprocessed red beans, which may be attributed to the changes of starch under higher pressure and through holding time, namely the fracture of some long-chain molecules into short-chain dextrin and the degradation of some long-chain molecules into soluble sugar. Analysis of variance indicates that among 13 experiments, the starch content of red beans processed under No.4 experimental condition (pressure: $0.8 \mathrm{MPa}$, holding time: 240s) and No.12 experimental condition (pressure: 0.6MPa, holding time: 320 s) significantly increased.

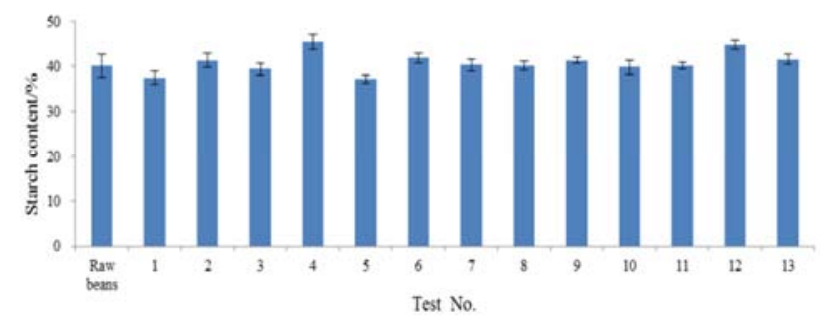

Figure 3. Starch content of explosion red beans and unprocessed beans.

\subsubsection{Effects of Steam Explosion Processing on the Crude Cellulose Content}

Table 5 shows the statistical analysis of crude cellulose content of unprocessed red beans and explosion red beans,, the average crude cellulose content of red beans decreased by $0.33 \%$, with insignificant change. Fig. 4 shows the changes of crude cellulose content of red beans under different experimental conditions, after the steam explosion processing 
under different conditions, the crude cellulose content of explosion red beans decreased by different levels. Analysis of variance indicates that compared with unprocessed red beans, the red beans processed under No. 4, 6, 8 and 11 experimental condition, the crude cellulose content significantly decreased, which indicates that the crude cellulose content of red beans would be significantly impacted under certain conditions of steam explosion processing.

Table 5. Variance anlaysis of crude cellulose content statistics of explosion red beans.

\begin{tabular}{llllll}
\hline Raw red beans & 4.39 & 4.78 & 4.61 & 3.850 & 0.057 \\
Explosion red beans & 3.72 & 4.78 & 4.28 & & \\
\hline
\end{tabular}

Note: ** means $\mathrm{P}<0.01$, very significant; * means $\mathrm{P}<0.05$, significant; $\mathrm{P}>0.05$, insignificant.

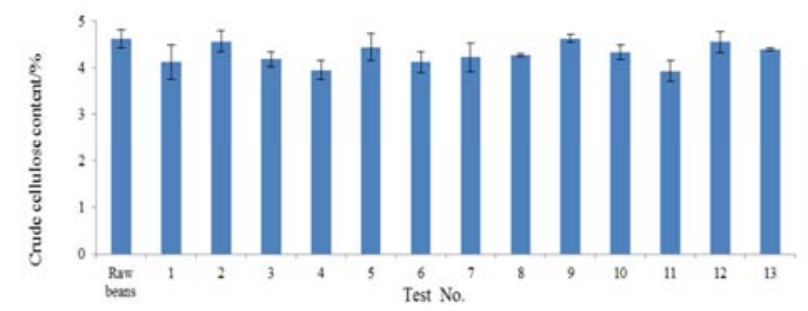

Figure 4. Crude cellulose content of explosion red beans and unprocessed beans.

\subsection{Effects of Steam Explosion Processing on the Puffing Ratio}

Table 6. Puffing ratio statistics of raw red beans and explosion red beans.

\begin{tabular}{llllll}
\hline Raw red beans & 100 & 100 & 100 & 68.974 & $0.000 * *$ \\
Explosion red beans & 166 & 231 & 195.4 & & \\
\hline
\end{tabular}

Note: ** means $\mathrm{P}<0.01$, very significant; * means $\mathrm{P}<0.05$, significant; $\mathrm{P}>0.05$, insignificant.

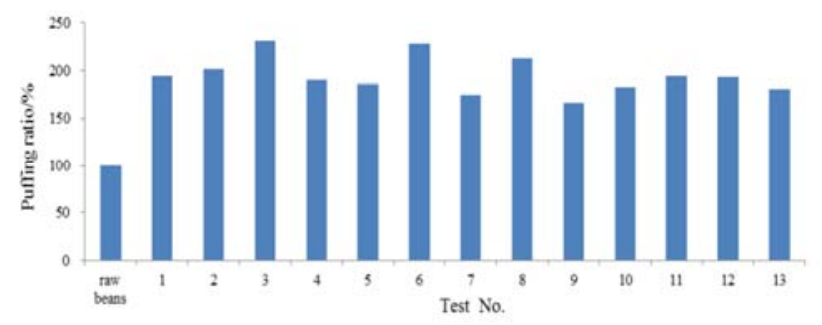

Figure 5. Puffing ratio of explosion red beans and unprocessed beans.

Assume the volume of unprocessed beans as 100 , after the steam explosion processing, the statistical analysis of red beans volume is as shown in Table 6. As it can be seen from Table 6, the average volume of red beans significantly increased, the average volume was $195.4 \%$ of the unprocessed red beans, and the smallest volume was $166 \%$ of the unprocessed red bean. If the puffing ratio statistics (see Table 6) of red beans under different experimental conditions were examined and through analysis of variance, the volume increase of red beans under each steam explosion condition was found to be very significant, which indicates that the steam explosion processing has a significant effect on the puffing ratio of red beans.

\subsection{Effects of Steam Explosion Processing on the Texture Properties of Red Beans}

\subsubsection{Effects of Steam Explosion Processing on the Hardness of Red Beans}

Table 7. Hardness statistics of explosion red beans and unprocessed red beans.

\begin{tabular}{llllll}
\hline Raw red beans & 43.7 & 106.9 & 74.5 & 43.657 & $0.000^{* *}$ \\
Explosion red beans & 10.0 & 124.1 & 52.5 & & \\
\hline
\end{tabular}

Note: ** means $\mathrm{P}<0.01$, very significant; * means $\mathrm{P}<0.05$, significant; $\mathrm{P}>0.05$, insignificant.

Table 7 shows the statistical analysis of hardness of unprocessed red beans and explosion red beans. As it can be seen from Table 7, the average hardness of explosion red beans decreased by $22.0 \mathrm{~N}$, with a very significant change. According to the hardness change of red beans under different experimental conditions (see Fig. 6), after the steam explosion processing, the hardness of red beans reduced by different levels, with a very significant change, particularly the hardness of red beans under No. 4, 11 experimental condition reduced by the highest levels. This indicates that the steam explosion processing may significantly reduce the hardness of red beans.

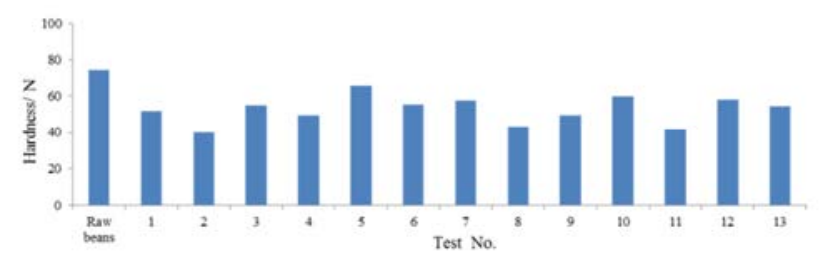

Figure 6. Hardness of explosion red beans and unprocessed beans.

\subsubsection{Effects of Steam Explosion Processing on the Springiness of Red Beans}

Table 8. Springiness statistics of the explosion red beans.

\begin{tabular}{llllll}
\hline Raw red beans & 0.10 & 3.66 & 0.43 & 1.686 & 0.194 \\
Explosion red beans & 0.06 & 7.00 & 0.58 & & \\
\hline
\end{tabular}

Note: ** means $\mathrm{P}<0.01$, very significant; * means $\mathrm{P}<0.05$, significant; $\mathrm{P}>0.05$, insignificant.

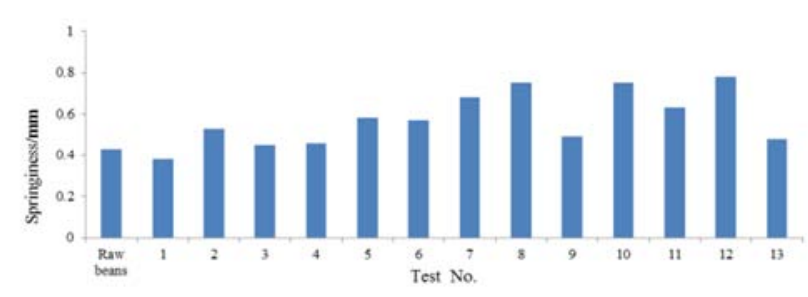

Figure 7. Springiness of explosion red beans and unprocessed beans.

Table 8 shows the statistical analysis of springiness of unprocessed red beans and explosion red beans, as it can be seen from Table 8, the average springiness of explosion red beans increased, but with an insignificant change. Fig. 7 
shows the springiness change of red beans under different experimental conditions. Fig. 7 suggests that under most experimental conditions, after the steam explosion processing, the springiness of red beans increased, but with an insignificant change. Therefore, it can be understood that the steam explosion processing has no significant effect on the springiness of red beans.

\subsubsection{Effects of Steam Explosion Processing on the Chewiness of Red Beans}

Table 9. Chewiness statistics of the explosion red beans.

\begin{tabular}{llllll}
\hline Raw red beans & 0.11 & 5.64 & 1.81 & 2.918 & 0.088 \\
Explosion red beans & 0.03 & 43.83 & 2.88 & & \\
\hline
\end{tabular}

Note: ** means $\mathrm{P}<0.01$, very significant; * means $\mathrm{P}<0.05$, significant; $\mathrm{P}>0.05$, insignificant.

Table 9 presents the statistical analysis of chewiness of unprocessed red beans and explosion red beans, as it can be seen from Table 9, the chewiness of unprocessed red beans was smaller, after the steam explosion processing, it increased by $1.07 \mathrm{~mJ}$, but with an insignificant change. After the processing under different experimental conditions, the chewiness of red beans dramatically changed as shown in Fig. 8. Under No. 4, 11 and 12 experimental condition, the average chewiness of red beans reached 5.42, 4.0 and $3.49 \mathrm{~mJ}$, which was equivalent to $2.99,2.21$ and 1.93 times of the unprocessed beans respectively, with a very significant change.

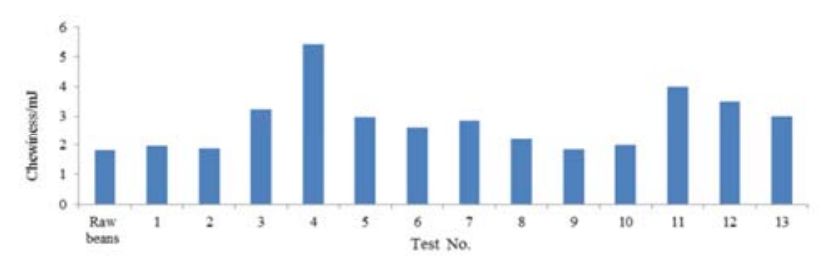

Figure 8. Chewiness of explosion red beans and unprocessed beans.

\section{Conclusion}

After the steam explosion processing, the crude protein, crude fat, starch or crude cellulose content of red beans experienced a small loss, which indicates that the steam explosion processing has no significant effect on the nutritive contents of red beans. After such processing, the average puffing ratio of red beans increased by $95.4 \%$, while the average hardness reduced by $22.0 \mathrm{~N}$, which indicates that the steam explosion processing has a very significant effect on the puffing ratio and hardness; in addition, the steam explosion processing may increase the average springiness and chewiness of red beans to some extent, but with an insignificant effect.

\section{References}

[1] Y. C. Zhang, W. X. Li, L. X. Huang. The research of starch properties of Adsuki bean [J]. Food Science, 2006, 27 (3):44-47.

[2] L. F. Wang. The safety and nutritiveness of puffed food [J]. Journal of Food Safety and Quality, 2008, (08):51-52.

[3] P. Wang, S. C. Ren, G. L. Wang. The nutritive characteristics and functional features of common food legumes [J]. Food Research and Development, 2009, 30 (12):171-174.

[4] T. B. Ren, X. Q. Ma, G. Z. Xum et al. The optimization of steam explosion preprocessing conditions through response surface methodology [J]. Transactions of the Chinese Society of Agricultural Engineering, 2011, 27 (9):282-286.

[5] H. Z. Chen, Z. H. Li. The new non-pollution stalk steam explosion technology and its application [J]. Journal of Cellulose Science and Technology, 2002, 10 (03):47-52.

[6] J. Yan, L. X. Feng. The research of steam explosion technology [J]. Modern Agricultural Science and Technology, 2009, (11):278-280.

[7] Baboukani B S, Vossoughi M, Alemzadeh I. Optimisation of dilute-acid pretreatment conditions for enhancement sugar recovery and enzymatic hydrolysis of wheat straw [J]. Biosystems Engineering, 2012, 111 (2): 166-174.

[8] Ozkal S G, Yener M E, Bayindirli L. Response surfaces of apricot kemel oil yield in supercritical carbon dioxide [J]. LWT-Food Science and Technology, 2005, 38 (6):611-616.

[9] Paul Koona, Valere Tatchago, Dorothy Malaa. Impregnated bags for safer storage of legume grains in West and Central Africa Original Research Article [J]. Journal of Stored Products Research, 2007, 43 (3):248-251.

[10] C. M. Mutungi, H. Affognon, A. W. Njoroge, D. Baributsa, L. L. Murdock. Storage of mung bean (Vigna radiata [L.] wilczek) and pigeonpea grains (Cajanus cajan [L.] Millsp) in hermetic triple-layer bags stops losses caused by Callosobruchus maculatus (F.) (Coleoptera: Bruchidae) [J]. Journal of Stored Products Research, 2014, 58 (6):39-47.

[11] C. H. Liu. The microwave vacuum puffing technology and quality of black currant fruit slices [D]. Harbin: Northeast Agricultural University, 2010.

[12] Z. Q. Zhang, S. B. Xiong, Y. Z. Li, et al. TPA of engineer reformed rice and sensory evaluation correlation analysis $[\mathrm{J}]$. Journal of The Chinese Cereals and Oils Association, 2011, 26 (10):1-5.

[13] J. Cao, J. W. Zhao, Y. Q. Tian, et al. Effects of extrusion process on the quality of preservation instant rice [J]. Journal of Food Science and Biotechnology, 2014, 33 (4):381-386. 